\title{
Processing of Wireless Signals to Preserve Wireline Network Resources.
}

\author{
Muriel Médard \\ Coordinated Science Laboratory \\ University of Illinois at Urbana-Champaign \\ Room 128, 1308 West Main Street \\ Urbana, IL 61801 \\ Medard@comm.csl.uiuc.edu
}

\begin{abstract}
In order increase capacity in the wireless domain, the signals from several distributed receivers may be combined. The combining of these signals typically requires transmission over a wireline network. In the wireline domain, signals may be transmitted to a single centralized processing node or may be processed in a distributed fashion at several nodes in the network. We compare, for white Gaussian noise channels, the capacity gains that can be obtained from distributed processing of wireline signals to the capacity gains obtained using maximum likelihood ratio combining at a single processing node. We find that, by using optimal detection techniques, the bandwidth requirements in the wireline domain can be significantly reduced without reducing the capacity in the wireless domain. These gains in capacity are achieved by eliminating transmission of redundant information in the wireline network.
\end{abstract}

\section{INTRODUCTION}

Wireless access to wireline networks is emerging as an important component of the drive towards ubiquitous communication services. Since wireless links suffer from more intrinsic limitations than wireline links, the emphasis has generally been on improving the rates available in the wireless domain. In particular, having several receivers yields significant gains in terms of rate for wireless communications. Thus, an attractive approach is to combine, over a wireline network, the signals of several receivers to obtain rate gains in the wireless network. An example of a simple form of such combining is soft-handoff in the IS-95 standard [9].

Little attention has been paid to how the use of several receivers affects resource management in the wireline domain. The main reason is that resources in the wireline domain generally do not suffer from the same drawbacks as wireless resources, for instance fading, power limitations and stringently limited bandwidth. However, wireline resources are not free and must be used judiciously. The purpose of this paper is to investigate how wireline resources may be preserved while obtaining all the benefits of combining the signals from several receivers. We consider capacity, i.e. maximum achievable rate, and show that, for a simple additive Gaussian noise (AWGN) channel model, distributed processing, which requires less use of bandwidth in the wireline domain, is equally as effective as centralized processing.

In Section II, we present the problem in terms of signals in the wireless and wireline domain. In Section III, we show that capacity for distributed and centralized processing is the same and show how such capacity may be achieved using optimal detection techniques. In Section IV, we present some numerical results illustrating the advantages of using distributed processing over centralized processing. The gains are in terms of spare capacity in the wireline domain. Finally, in Section V, we discuss our results and present directions for further research.

\section{PROBLEM STATEMENT.}

In order to increase capacity of wireless systems and to overcome the lack of reliability inherent to fading wireless links, we may use the signals received by several wireless access ports, each connected to an antenna or group of antennas, and combine the signals in the wireline domain. In this paper, we consider placing the output from several antennas, or groups of antennas, in the wireline domain to increase the rate available to wireless users. We a receiver each antenna or group of antennas attached to an access port to the wireline network. There are two options for processing, in the wireline domain, the streams from different access ports. The first option is to take the streams and combine them at a single processing node, as shown in Figure 1.a. If the access ports are not co-located, then the wireline infrastructure will have to support several streams concurrently. A second option, which is illustrated in Figure 1.b, is to have distributed processing among access ports. That option requires a single stream to travel over any portion of the wireline infrastructure. We do not consider here the issue of hardware cost comparisons between having a few centralized processing nodes and many distributed processing nodes. We only consider the issue of what data rates need to be sent in the wireline domain.

There has been much research in the area of optimal combining of antennas, or of selecting the best antenna. The capacity of multi-receiver networks with Gaussian noise has been considered in $[5,6,11]$. The use of multiple antennas has been considered also in fading environments for cellular communications [10] and for indoor wireless environments [2]. The use of several receivers has prompted space-time coding schemes for enhancing capacity [7]. A good overview of multi-receiver results in fading channels may be found in [1]. Note that we do not consider the interesting issue of multiple sender antennas [8]. All of the above work is concerned with selecting or combining signals from antennas 
but does not consider the networking aspects of such combinations.

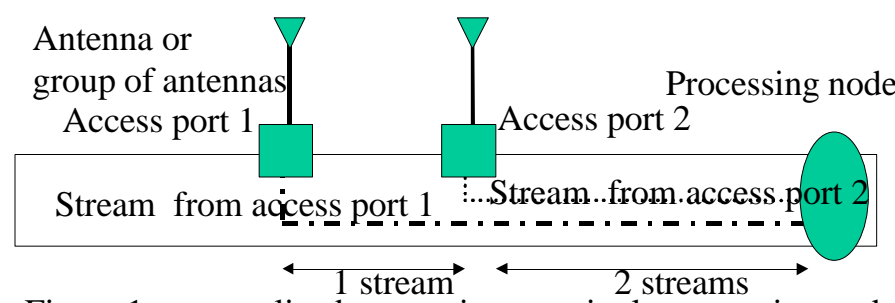

Figure 1.a: centralized processing at a single processing node.

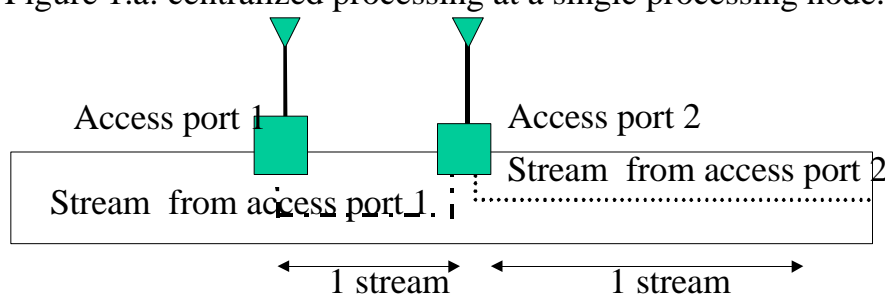

Figure 1.b: distributed processing at several nodes.

The approach we propose is to provide means for obtaining the capacity enhancement provided by multiple antennas while using the wireline resources efficiently. This question becomes important because the model of limited bandwidth in the wireless domain and unlimited bandwidth in the wireline domain is overly simplistic. Let us suppose that each user is received by, on average, two receivers, so that some diversity gains from multiple receivers are achieved. Let us suppose, at first approximation, that all streams in Figure 1 require roughly the same capacity. If the links at access ports are dedicated to carrying signals to and from processing nodes, then the wireline infrastructure will have to be built with double the capacity required if only one stream was present at any time. If access ports are connected to a network which bears other traffic, then, for some portion of the network before the processing node, each wireless user will require in the wireline domain roughly twice as much rate as it is actually getting. If more receivers are added, then even more rate will be used up in the wireline domain, with all the associated costs. In the case where the wireline domain does not have very large bandwidth, then sending streams from several access ports to a single processing node, as in Figure 1.a, may rapidly cause congestion. Thus, provisioning rate in the wireless domain can be hampered by the fact that multi-receiver schemes may congest the wireline link. Our discussion motivates us to consider the model of Figure 1.b and to pose the question of whether such a model can yield the same capacity as that of Figure 1.a.

\section{CAPACITY OF DISTRIBUTED VS. CENTRALIZED PROCESSING}

We may illustrate our approach with a simple example. Let us consider just three wireless receivers. We first discuss coding in the wireless domain to accommodate the use of one or many of the access nodes in the wireline domain. Each receiver receives the signal, $\mathrm{X}$, sent over bandwidth $\mathrm{W}$ by a unique mobile user with average energy constraint $\mathrm{E}$. The signal is corrupted only by AWGN, which is independent for the three receivers and has spectral density $\mathrm{N}_{0}$. We assume the noise has the same energy for the two receivers but is independent at the receivers. In that case, using well-known results for systems with multiple antennas, we know that capacity, i.e. maximum achievable rate, can be achieved by maximum likelihood ratio (MLR) combining. In this case, capacity can be obtained as

$\mathrm{R}=\frac{1}{2} \log \left(1+\frac{3 E}{W N_{0}}\right)$ bits/s

and appropriate coding allows this rate to be approached arbitrarily well. In the case where only one of the receivers is available, however, a simple WGN-style code, which yielded a rate near capacity when the two users were present, will no longer yield the new capacity, which is

$\mathrm{R}^{\prime}=\frac{1}{2} \log \left(1+\frac{E}{W N_{0}}\right)$ bits/s.

A multi-resolution code, such as would be used for achieving capacity in broadcast channels, can be used to achieve rates in some region which has $\mathrm{R}$ and $\mathrm{R}$ ' as endpoints [3, 4]. Note that, when the SNR is very high, then both rates are basically equal.

The above example shows that achievable rates with multiple antennas depend on whether we are considering that all antennas are available or that some of the antennas may or may not be available. While we have simply looked at the rate available to one user, we also must consider the number of users. Let us now consider that, instead of allowing each antenna receiver to place the entire signal it received onto the wireline link through its access port, it only places that portion which is a new contribution. Consider again our simple example with three receivers. If each receiver simply transmits in the wireline domain independently of whether the other receiver was also active, then each receiver places $\mathrm{R}^{\prime}$ in the optical domain. If the SNR is high, then 2R', basically three times the rate necessary to transmit $R$, is used. Thus, the number of users is divided by 3 without providing a real difference in rate to each user. A simple technique is to allow only one active receiver to send, which corresponds to selecting the best receiver.

In order to obtain the benefit from having several receivers, it is not always sufficient to select just one. In particular, in the case of low-rate users, who may not have high SNRs, reaping the benefit of having several receivers will not be possible unless each receiver places its contribution in the wireline domain. For low rate users, having three receivers instead of one yields important benefits. Thus, using the same method for users at different rates may not be efficient. For low-rate users, having each receiver transmit is close to optimal. Note 
that these low-rate users may be very important and therefore cannot be neglected. Figure 2 shows the set-up we consider: 3 access ports, each of which receives its signal from a single antenna. Each antenna receives the same user over an AWGN channel. All channels have the same power AWGN and the noises, denoted $\mathrm{N}_{\mathrm{i}}$ for receiver $\mathrm{i}$, are IID. Let us consider that access port 1 sends signal $X_{1}$ to access port 2 , where $X_{1}$ is the maximum likelihood (ML) estimate of $\mathrm{X}$ from $\mathrm{Y}$. Because of our AWGN channel $X_{1}$ is also the minimum mean square error (MMSE) estimate and the linear least square error (LLSE) estimate of $X$ from $Y_{1}$. We have that $X_{1}=Y_{1} \frac{E}{E+N_{0} W}$. The error of this estimate has variance $\sigma_{1}^{2}=\frac{E N_{0} W}{E+N_{0} W}$. Note that transmission of $\mathrm{X}_{1}$ in the wireline domain is equivalent to transmission of $Y_{1}$ in the wireline domain. At access port 2, we generate the $M L$ estimate $X_{2}$ of $X$ from $Y_{2}$ and $X_{1}$, i.e. $Y_{1}$. The error on this estimate has variance

$\sigma_{2}^{2}=\sigma_{1}^{2}-\frac{\sigma_{1}^{4}}{\sigma_{1}^{2}+N_{0} W}=\frac{E N_{0} W}{2 E+N_{0} W}$ and is Gaussian. Note that the variance of the error is the same as obtained by MLR combining of $Y_{1}, Y_{2}$. Next, if we send the estimate $X_{2}$ of $X$ to port 3, we have an estimate $\mathrm{X}_{3}$ obtained from $\mathrm{Y}_{3}$ and $\mathrm{X}_{2}$, with error variance $\sigma_{3}^{2}=\sigma_{2}^{2}-\frac{\sigma_{2}^{4}}{\sigma_{2}^{2}+N_{0} W}=\frac{E N_{0} W}{3 E+N_{0} W}$, which is the same as that obtained by MLR combining of $\mathrm{Y}_{1}, \mathrm{Y}_{2}$ and $\mathrm{Y}_{3}$. The error is again Gaussian. Thus, sending only one estimate down the link between any two access ports yields the same estimate that would be yielded by combining all three Ys at a central processing node.

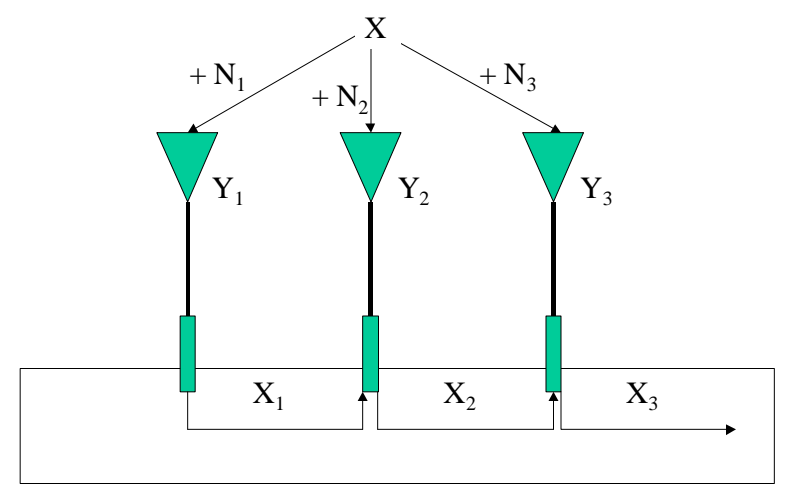

Figure 2: Distributed processing over three nodes

Moreover, $\mathrm{I}\left(\mathrm{X} ;\left(\mathrm{Y}_{1}, \mathrm{Y}_{2}, \mathrm{Y}_{3}\right)\right)=\mathrm{h}(\mathrm{X})-\mathrm{h}\left(\mathrm{X} \mid \mathrm{Y}_{1}, \mathrm{Y}_{2}, \mathrm{Y}_{3}\right)$. Thus, $\mathrm{I}\left(\mathrm{X} ;\left(\mathrm{Y}_{1}, \mathrm{Y}_{2}, \mathrm{Y}_{3}\right)\right)$ is maximized by setting $\mathrm{X}$ to be Gaussian. In that case, $\mathrm{h}\left(\mathrm{X} \mid \mathrm{Y}_{1}, \mathrm{Y}_{2}, \mathrm{Y}_{3}\right)$ depends solely on the error of the estimate on $\mathrm{X}$ from $\left(\mathrm{Y}_{1}, \mathrm{Y}_{2}, \mathrm{Y}_{3}\right)$ in the following manner:

$\mathrm{h}(\mathrm{X} \mid \mathrm{Y} 1, \mathrm{Y} 2, \mathrm{Y} 3)=\frac{1}{2} \ln \left(2 \pi \sigma_{3}^{2}\right)$. Hence, the same maximum mutual information is obtained by using MLR combining and distributed processing. Thus, the model of Figure 1.b yields the same maximum mutual information as that of Figure 1.a while reducing significantly the amount of bandwidth required in the wireline link. Our approach can clearly be extended to an arbitrary number of antennas.

We have shown that the same maximum mutual information is obtained with MLR or distributed processing. However, if we are interested in capacity, we must also consider what type of coding, if any, can be used to achieve a rate which is arbitrarily close to the time average maximum mutual information with distributed processing. Fortunately, the channel model we obtain both for MLR and distributed estimation is the following:

$\mathrm{Y}=\mathrm{X}+\mathrm{N}$, where $\mathrm{N}$ has variance $\sigma_{3}^{2}$. Thus, a random code yielding a WGN signal for $\mathrm{X}$ will achieve capacity for either MLR or distributed estimation. Hence, the coding performed in the wireless domain is not affected by how estimation is performed in the wireline domain.

\section{NUMERICAL RESULTS}

We can illustrate the benefits of distributed processing versus centralized processing. Over the wireline, we consider that communications have very low noise, so we consider that the rate needed to transmit data is the data rate. The first measure we consider is the ratio of the maximum required rates. In terms of Figure 2, the benefit is the ratio between the wireline domain rate required to transmit $\mathrm{X}_{3}$ and that required to transmit $Y_{1}, Y_{2}$ and $Y_{3}$. For $n$ receivers, each with the same $\mathrm{SNR}$, this benefit may be expressed as:

$\mathrm{b}(\mathrm{n})=\frac{\ln (1+\mathrm{n} \mathrm{SNR})}{\mathrm{n} \ln (1+\mathrm{SNR})}$

Another measure is the ratio of the total rates. Each total rate is the sum, over all links, of the rate used over each link. This expression is:

$b^{\prime}(n)=\frac{\sum_{i=1}^{n} \ln (1+i S N R)}{\sum_{i=1}^{n} i \ln (1+S N R)}$

Figures 3 and 4 show plots of $b(n)$ and $b^{\prime}(n)$ for $n$ from 1 to 8 and SNRs ranging from 1 to 10 , with the curves increasing with the SNR parametrization. 
We have shown that, for mutually independent AWGN channels with known noise variance, obtaining the benefits from several antennas may not require the overhead in wireline bandwidth associated with conventional techniques. Several research issues stem from this fact. The first one is how well coding designed for a particular set of antennas will work when a different set of antennas, possibly a subset of the original set of antennas, is available. Moreover, we assumed that the channel is AWGN and that the noise power at each antenna is known a priori. In the case of time-varying channels rather than AWGN channels, when estimation of a different channel may be required at each antenna, does the successive transmission of estimates yield equal results to central processing and, if not, how different are those results? When we have multiple access at the wireless access nodes, how do our estimation arguments extend? Is it worthwhile for the receivers to transmit the entirety of what they have received? Such a policy, as we have seen, is beneficial for low-rate users, but may be marginally so for high-rate users. Do the receivers need to separate the contribution of each user and follow different policies for different rate users?
[1] E Biglieri, J. Proakis, S. Shamai, "Fading Channels: InformationTheoretic and Communication Aspects", IEEE Trans. Information Theory, vol. 44, no. 6, Oct. 1998.

[2] C.-N. Chuah, D. Tse, J. Kahn, "Capacity of Multi-Antenna Array Systems in Indoor Wireless Environments", Globecom 98, Sydney, Australia.

[3] T.M. Cover, "Broadcast Channels", IEEE Trans. on Info. Theory, IT-18, pp. 2-14, 1972.

[4] T.M. Cover, "An Achievable Rate Region for the Broadcast Channel", IEEE Trans. on Info. Theory, IT-21, 1975, pp. 399-404.

[5] S.V. Hanly, Information Capacity of Radio Networks, Ph.D. Thesis, University of Cambridge, August 1993.

[6] S.V. Hanly, P. Whiting, "Information-Theoretic Capacity of MultiReceiver Networks", Telecommunication Systems, 1, 1993, pp. 1-42.

[7] B. Hochwald, T.L. Marzetta, "Space-Time Modulation Scheme for Unknown Rayleigh Fading Environments", $36^{\text {th }}$ Allerton Conference on Comm., Control and Computing, University of Illinois, Urbana, IL 1998. [8] A. Narula, M.T. Trott, G. W. Wornell, "Information-theoretic Analysis of Multiple-antenna transmission diversity for fading channels", submitted to IEEE Transactions on Info. Theory.

[9] Qualcomm, IS-95-A: the cdmaOne Standard (CDMA-255) Student Guide, Qualcomm 1997.

[10] S. Shamai, A.D. Wyner, "Information Theoretic Considerations for Symmetric, Cellular Multiple-Access Fading Channels - Parts I and II", IEEE Trans. on Info. Theory, vol. 43, no. 6, Nov. 1997, pp. 1877-1911. [11] I.E. Telatar, "Capacity of Multi-Antenna Gaussian Channels", AT\&T Bell Laboratories, Technical Memorandum, 1995.

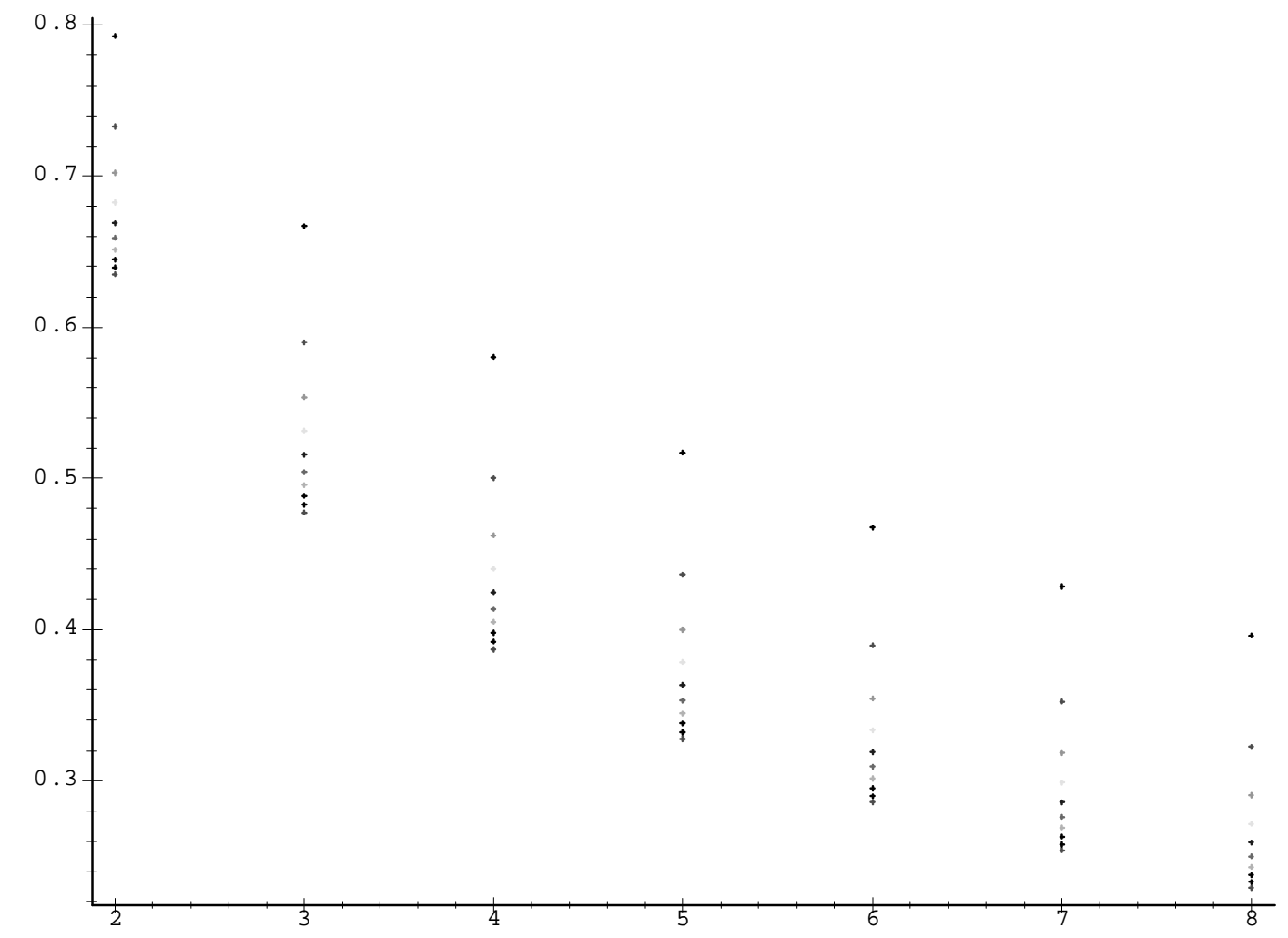

Figure 3: First measure of benefit of distributed processing. The plot is of $b(n)$ versus $n$ for SNRs of 1 through 10, from bottom to top, respectively. 


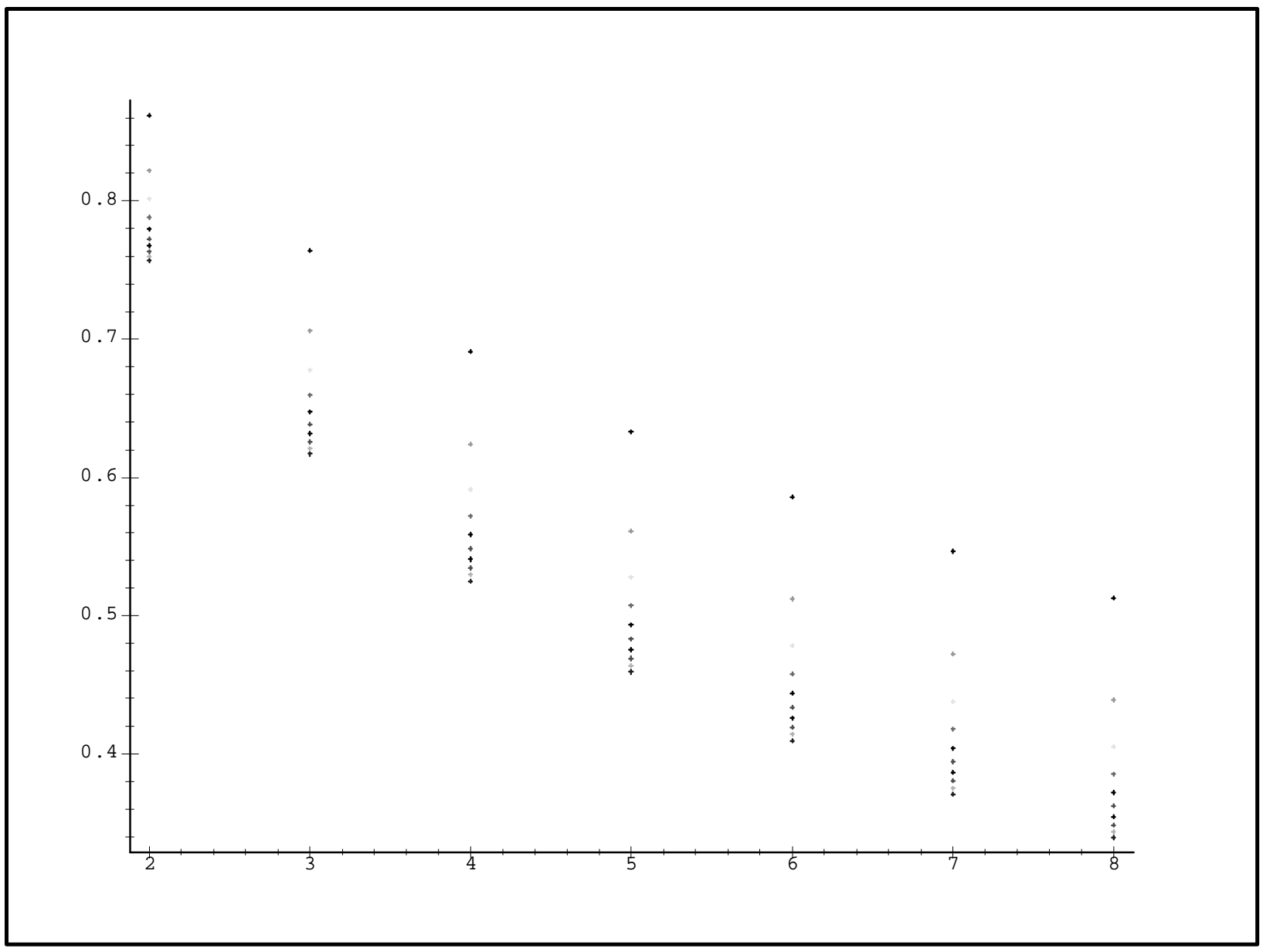

Figure 4: Second measure of benefit of distributed processing. The plot is of $b^{\prime}(n)$ versus $n$ for SNRs of 1 through 10, from bottom to top, respectively. 